\title{
A New MATLAB based Microstrip Filter Design Tool
}

\author{
Shreyas Rao ${ }^{\mathrm{a}}$, Raghuvir Tomar ${ }^{\mathrm{b}^{*}}$ \\ ${ }^{a}$ School of Engineering, VIT University Chennai Campus, Vandalur-Kelambakkam Road, Chennai 600127, \\ India \\ ${ }^{b}$ Department of Electronics and Communication Engineering, The LNM Institute of Information Technology, \\ Gram Rupa-Ki-Nangal, Post-Sumel, Via Jamdoli, Jaipur 302031, India
}

Received: 16 December 2016; Accepted: 08 June 2017; Published: 08 September 2017

\begin{abstract}
This paper presents a new MATLAB-based microstrip filter design tool. Stepped-impedance resonator lowpass filter, parallel-coupled bandpass filter, and end-coupled bandpass filter are included. Circuit-theory based closed-form mathematical expressions are used, instead of rigorous electromagnetic analyses. This results in significant reduction in analytical effort, without substantially affecting the accuracy. The results are verified against results obtained using commercially available Ansoft simulation tool. The agreement between the two sets of results is good enough for all practical purposes.
\end{abstract}

Index Terms: Microstrip, Filter, RF, MIC, Ansoft, MATLAB.

(C) 2017 Published by MECS Publisher. Selection and/or peer review under responsibility of the Research Association of Modern Education and Computer Science

\section{Introduction}

Filters play a major role in designing modern communication systems. In radio frequency (RF)/microwave frequency band, typically between $300 \mathrm{MHz}$ and $20 \mathrm{GHz}$, microstrip filters are a better choice over lumpedcomponent filters because of microstrip filters' small size, their compact design, and their low-cost reliable manufacturing.

The analysis and synthesis of microstrip filters is a constantly evolving area of research [1]-[17]. Traditionally, such filters were designed using extensive electromagnetic-theory based analyses. However, the mathematical complexity and computational effort involved were high. Consequently, the use of 'modified' circuit theory approach is also being paid serious attention to, since the use of this approach significantly

* Corresponding author. Tel.: +919001708555; fax: +911412689014

E-mail address: tomar_lnmiit@gmx.com,rtomar@lnmiit.ac.in,raoshreyas96@yahoo.in 
reduces the analytical rigor involved, without appreciably compromising the accuracy of results, especially in the lower portion of the RF/microwave frequency band.

In the present work, we have used the circuit-theory approach to develop a new MATLAB-based design/analysis tool for three most popular microstrip filters, namely, stepped-impedance resonator lowpass filter, parallel-coupled bandpass filter, and end-coupled bandpass filter. The accuracy of tool has been verified using several design examples, against data obtained using commercially available Ansoft simulator. The agreement between the two sets of data is good enough for all practical purposes.

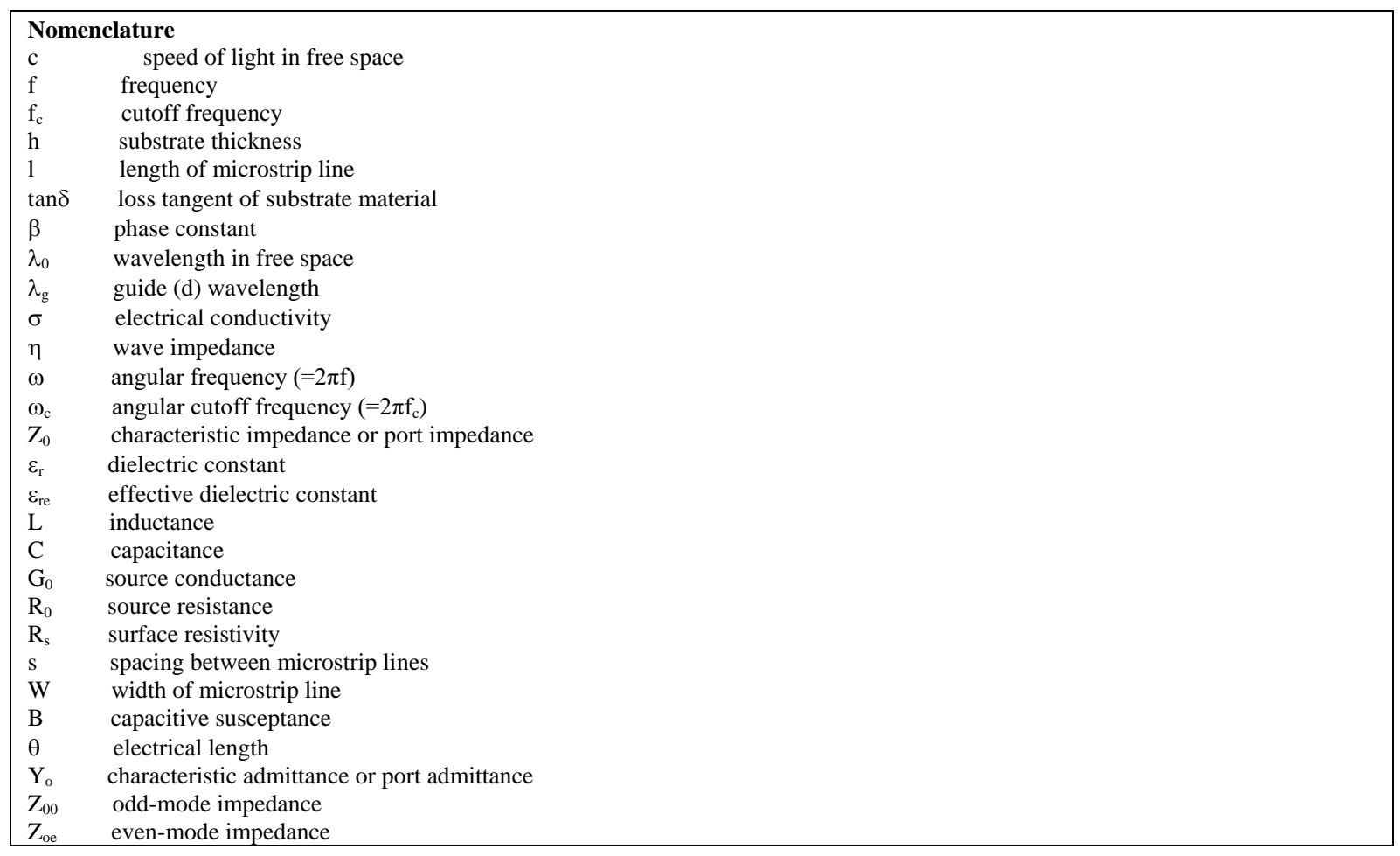

\section{Microstrip Transmission Line}

The microstrip transmission line, as shown in Fig. 1, consists essentially of a dielectric substrate with dielectric constant $\varepsilon_{\mathrm{r}}$ and substrate thickness (also called 'substrate height') h. The bottom surface of the substrate is fully metallized to serve as a 'ground plane'. The top surface is bare, except for a conducting metallic strip of width $\mathrm{W}$, length 1 , and thickness $\mathrm{t}$. The value of $\mathrm{t}$ is typically between $4 \mu \mathrm{m}$ and $16 \mu \mathrm{m}$. The values of $\mathrm{W}, \mathrm{h}$, and 1 can range from a few mils to hundreds of mils where one mil is defined to be equal to 0.001 inch. Some commonly used values of the substrate dielectric constant are $\varepsilon_{\mathrm{r}}=2.2$ (Rogers Corporation substrate RT5080), 4.4 (FR4 substrate), 6.0 (Rogers Corporation substrate RT6010), 9.8 (Alumina substrate), and 12.9 (Gallium Arsenide substrate). 


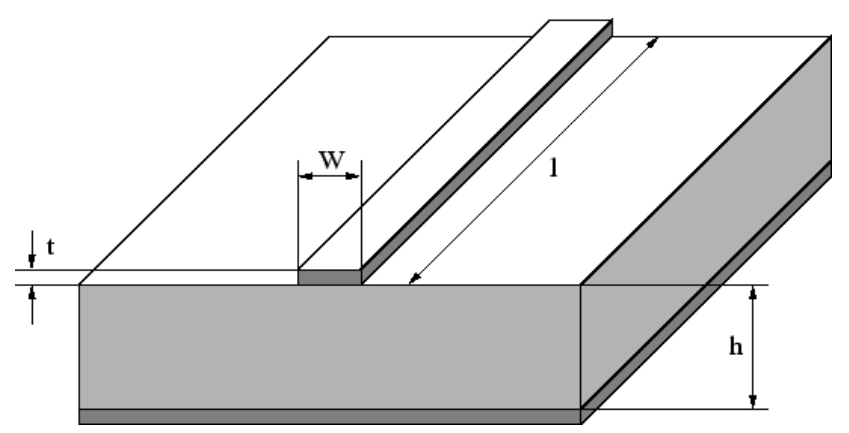

Fig.1. The schematic of a microstrip line

\subsection{Performance Parameters}

The two most important performance parameters of a microstrip line are its effective dielectric constant $\varepsilon_{\text {re }}$, and its characteristic impedance $Z_{0}$. The effective dielectric constant can be interpreted as the dielectric constant of a homogeneous medium that equivalently replaces the air-dielectric combination seen in the microstrip. The knowledge of effective dielectric constant helps in predicting the phase-velocity and phase-shift of the signal traveling inside the microstrip. The characteristic impedance can be interpreted as the impedance that the microstrip presents to the external world under matched conditions. The knowledge of characteristic impedance helps in ensuring the best possible transfer of power into and out of the microstrip.

The semi-empirically derived formulas for the effective dielectric constant (taken from [4]) follow.

For $W / h \leq 1$

$$
\varepsilon_{r e}=\frac{\varepsilon_{r}+1}{2}+\frac{\varepsilon_{r}-1}{2}\left[\left(1+12 \frac{h}{W}\right)^{-0.5}+0.04\left(1-\frac{W}{h}\right)^{2}\right]
$$

For $W / h \geq 1$

$$
\varepsilon_{r e}=\frac{\varepsilon_{r}+1}{2}+\frac{\varepsilon_{r}-1}{2}\left(1+12 \frac{h}{W}\right)^{-0.5}
$$

Note that depending on the range in which the value of the aspect ratio $\mathrm{W} / \mathrm{h}$ falls, the formula to be used is somewhat different.

The formulas for the characteristic impedance follow.

For $W / h \leq 1$

$$
Z_{o}=\frac{\eta}{2 \pi \sqrt{\varepsilon_{r e}}} \ln \left(\frac{8 h}{W}+0.25 \frac{W}{h}\right)
$$

For

$W / h \geq 1$ 


$$
Z_{o}=\frac{\eta}{\sqrt{\varepsilon_{r e}}}\left[\frac{W}{h}+1.393+0.67 \ln \left(\frac{W}{h}+1.44\right)\right]^{-1}
$$

where $\eta=120 \pi \Omega$ is the wave impedance in free space. The effects of strip thickness $t$ are assumed negligible in (1) and (2). This is quite justified in view of the fact that it is generally very small compared to h. However, in case it is large enough, modified versions of (1) and (2) are available in literature [7].

The purpose of (1) and (2) is to analyze a given microstrip, that is, to determine the values of $\varepsilon_{\mathrm{re}}$ and $\mathrm{Z}_{0}$ for a given set of values of $\mathrm{W}, \mathrm{h}$, and $\varepsilon_{\mathrm{r}}$. The reverse process, namely, the designing of the microstrip, involves calculating $\mathrm{W}$ for a given set of values of $\mathrm{h}, \varepsilon_{\mathrm{r}}$, and $\mathrm{Z}_{0}$. This is achieved using the following set of equations.

For $W / h \leq 2$

$$
\frac{W}{h}=\frac{8 e^{A}}{e^{2 A}-2}
$$

For $W / h \geq 2$

$$
\frac{W}{h}=\frac{2}{\pi}\left[B-1-\ln (2 B-1)+\frac{\varepsilon_{r}-1}{2 \varepsilon_{r}}\left[\ln (B-1)+0.39+\frac{0.61}{\varepsilon_{r}}\right]\right]
$$

where

$$
\begin{aligned}
& A=\frac{Z_{o}}{60} \sqrt{\frac{\varepsilon_{r}+1}{2}}+\frac{\varepsilon_{r}-1}{\varepsilon_{r}+1}\left(0.23+\frac{0.11}{\varepsilon_{r}}\right) \\
& B=\frac{377 \pi}{2 Z_{o} \sqrt{\varepsilon_{r}}}
\end{aligned}
$$

The parameters A and B given by (4) and (5) are local parameters and are meant to be used only in (3). They should not be confused with ABCD parameters of a two-port network discussed in sub-sections 2.2, 2.3, and 2.4.

The wavelength $\lambda_{\mathrm{g}}$ of the signal propagating inside the microstrip, called guide wavelength or guided wavelength is given by

$$
\lambda_{g}=\frac{\lambda_{o}}{\sqrt{\varepsilon_{r e}}}
$$

where the free-space wavelength $\lambda_{\mathrm{o}}$ is given by 


$$
\lambda_{o}=\frac{c}{f}
$$

In (7), $\mathrm{c}$ denotes the speed of light in vacuum, and $\mathrm{f}$ denotes the operating frequency in $\mathrm{Hz}$. The approximate value of $\mathrm{c}$ is $300,000,000 \mathrm{~m} / \mathrm{s}$.

The phase-velocity $\mathrm{v}_{\mathrm{p}}$ of the signal propagating inside the microstrip is given by the following equation.

$$
v_{p}=\frac{c}{\sqrt{\varepsilon_{r e}}}
$$

The phase-constant, $\beta$, of the signal traveling inside the microstrip (representing phase-shift introduced in signal per unit length) is given by the following equation.

$$
\beta=\frac{2 \pi}{\lambda_{g}}
$$

The complex propagation constant $\gamma$ is written as follows.

$$
\gamma=\alpha+j \beta
$$

where $\alpha$ is known as the attenuation-constant (measured in $\mathrm{dB} / \mathrm{m}$ or Neper $/ \mathrm{m}$ ) and $\beta$, as already mentioned, is known as the phase-constant (measured in radians $/ \mathrm{m}$ or degrees $/ \mathrm{m}$ ). The equation used for calculating the attenuation constant follows.

$$
\alpha=\alpha_{c}+\alpha_{d}
$$

where $\alpha_{c}$ represents the conductor loss (loss due to finite electrical conductivity of the metal used) and $\alpha_{d}$ represents the dielectric loss (loss due to dielectric material's lossy behavior). The equation for $\alpha_{c}$ follows.

$$
\alpha_{c}=\frac{R_{s}}{Z_{0} W}
$$

where the surface resistivity of the conductor, $R_{s}$, is given by

$$
R_{s}=\sqrt{\omega \mu_{0}} / \sqrt{2 \sigma}
$$

In (13), $\omega=2 \pi f$ is the angular frequency involved, $\sigma$ is the electrical conductivity of the metal used, and $\mu_{0}$ is the permeability of the metal used (assumed to be the same as that of air). Note that (12) yields the $\alpha_{c}$ value in $\mathrm{Np} / \mathrm{m}$ which needs to be multiplied by 8.6859 (approximately) to get the result in $\mathrm{dB} / \mathrm{m}$.

The equation for $\alpha_{d}$ follows. 


$$
\alpha_{d}=\frac{\beta \varepsilon_{r}\left(\varepsilon_{r e}-1\right) \tan \delta}{2 \sqrt{\varepsilon_{r e}}\left(\varepsilon_{r}-1\right)}
$$

where the result is again in $\mathrm{Np} / \mathrm{m}$ and needs to be converted to $\mathrm{dB} / \mathrm{m}$. In (14), tan $\delta$ is the loss-tangent of the substrate material whose value is given in the data sheet of the substrate material.

\subsection{Two-Port Representation}

For circuit-analysis purposes, it is customary to treat the microstrip as a two-port network as depicted in Fig. 2. The input variables (at port 1) are defined to be the input voltage $V_{1}$ and the input current $I_{1}$. The output variables at (port 2) are defined to be the output voltage $\mathrm{V}_{2}$ and the output current $\mathrm{I}_{2}$.

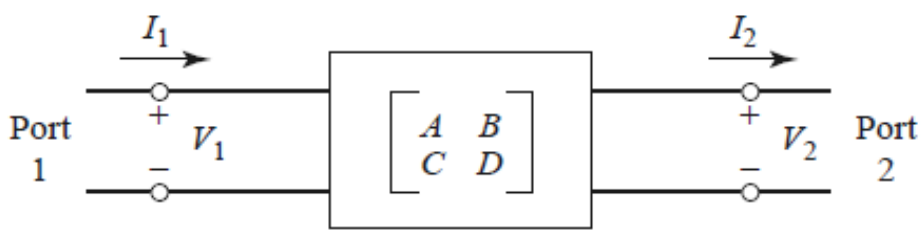

Fig.2. Two-port representation of a microstrip line

The ABCD parameters (also known as transmission parameters) of a two-port network are defined by the following equations (using the voltage polarities and current directions shown in Fig. 2).

$$
\begin{aligned}
& V_{1}=A V_{2}+B I_{2} \\
& I_{1}=C V_{2}+D I_{2}
\end{aligned}
$$

Another method used (especially at high frequencies) is to represent a two-port network in terms of incident and reflected signals at port 1 (that is, $a_{1}$ and $b_{1}$ ) and the same at port 2 (that is, $a_{2}$ and $b_{2}$ ). This is shown in Fig. 3. In this case, we define four scattering parameters, also known as $S$-parameters, $S_{11}, S_{12}, S_{21}$, and $S_{22}$.

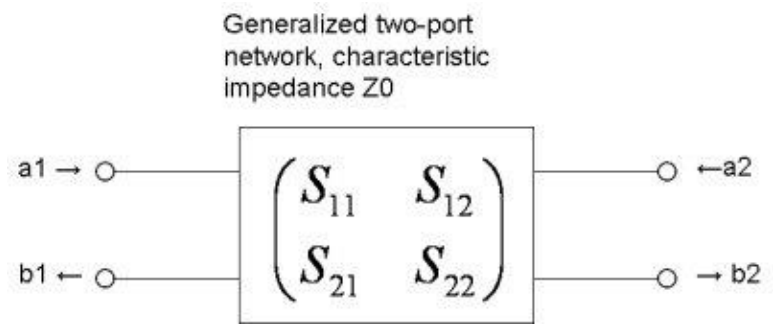

Fig.3. Scattering-parameter representation of a microstrip line

The equations for defining the S-parameters follow.

$$
b_{1}=S_{11} a_{1}+S_{12} a_{2}
$$


$b_{2}=S_{21} a_{1}+S_{22} a_{2}$

The equations for computing the S-parameters, from a given set of ABCD-parameter values, are given below.

$$
\begin{aligned}
S_{11}= & \frac{A+\frac{B}{Z_{0}}-C Z_{0}-D}{A+\frac{B}{Z_{0}}+C Z_{0}+D} \\
S_{12}= & \frac{2(A D-B C)}{A+\frac{B}{Z_{0}}+C Z_{0}+D} \\
S_{21}= & \frac{2}{A+\frac{B}{Z_{0}}+C Z_{0}+D} \\
S_{22}= & \frac{-A+\frac{B}{Z_{0}}-C Z_{0}+D}{A+\frac{B}{Z_{0}}+C Z_{0}+D}
\end{aligned}
$$

Note that for reciprocal networks like microstrip lines,

$$
A D-B C=1
$$

and hence

$$
S_{12}=S_{21}
$$

and

$$
S_{11}=S_{22}
$$

The insertion loss (IL) of the two-port network is defined by the following equation.

$$
I L(d B)=20 \log (X)
$$

where $\mathrm{X}$ is the magnitude of the transmission coefficient $\mathrm{T}$. In case of microstrip, $\mathrm{T}$ is defined to be equal to $\mathrm{S}_{12}$ (or $\mathrm{S}_{21}$ ). Hence, we write

$$
X=\left|S_{12}\right|=\left|S_{21}\right|
$$


The input return loss $\mathrm{RL}_{\text {input }}$ and output return loss $\mathrm{RL}_{\text {output }}$ are defined by the following equations.

$$
\begin{aligned}
& R L_{\text {inрu}}(d B)=-20 \log \left\{\left|S_{11}\right|\right\} \\
& R L_{\text {outpu }}(d B)=-20 \log \left\{\left|S_{22}\right|\right\}
\end{aligned}
$$

where $S_{11}$ is known as the input reflection coefficient and $S_{22}$ is known as the output reflection coefficient.

\subsection{ABCD Parameters of a Single Microstrip}

For a single microstrip line shown in Fig. 1 , with characteristic impedance $Z_{0}$, attenuation constant $\alpha$, phaseconstant $\beta$, and physical length 1 , the equations for calculating the ABCD parameters are given below. These equations include the effects of losses in the line and, if necessary, can be appropriately modified [4] for a lossless line by putting $\alpha=0$ and, hence, $\gamma=\mathrm{j} \beta$.

$$
\begin{aligned}
& A=\cosh (\gamma) \\
& B=Z_{0} \sinh (\gamma) \\
& C=\frac{1}{Z_{0}} \sinh (\gamma) \\
& D=\cosh (\gamma)
\end{aligned}
$$

Once the ABCD parameters are known, one can use (19)-(22) to compute the S-parameters.

\subsection{ABCD Parameters of a Chain of Cascaded Microstrips}

For a chain of cascaded microstrips (that is, the output of one microstrip feeding the input of the next microstrip, and so on), the overall ABCD-matrix can be calculated by multiplying the individual ABCDmatrices of the various microstrips in the correct sequence [4]. Once the overall ABCD parameters are known, one can use (19)-(22) to compute the overall S-parameters of the chain under consideration.

\section{Filter Design using lumped components}

The lumped-component based method of designing electronic filters is primarily meant for low frequencies. However, the theory involved remains highly relevant to microwave filters too, and will hence be summarized below.

\subsection{Lowpass Filter (LPF) Design}

As graphically shown in Fig. 4 (taken from [7]), a lowpass filter (LPF) is typically designed to have either a maximally-flat response (also known as Butterworth response or binomial response) or an equal-ripple response (also known as Chebyshev response). In the maximally-flat response, the passband has no ripples. In the equal-ripple response, the passband has ripples. The rate of rise of Insertion Loss with frequency is relatively higher in the case of equal-ripple response. 


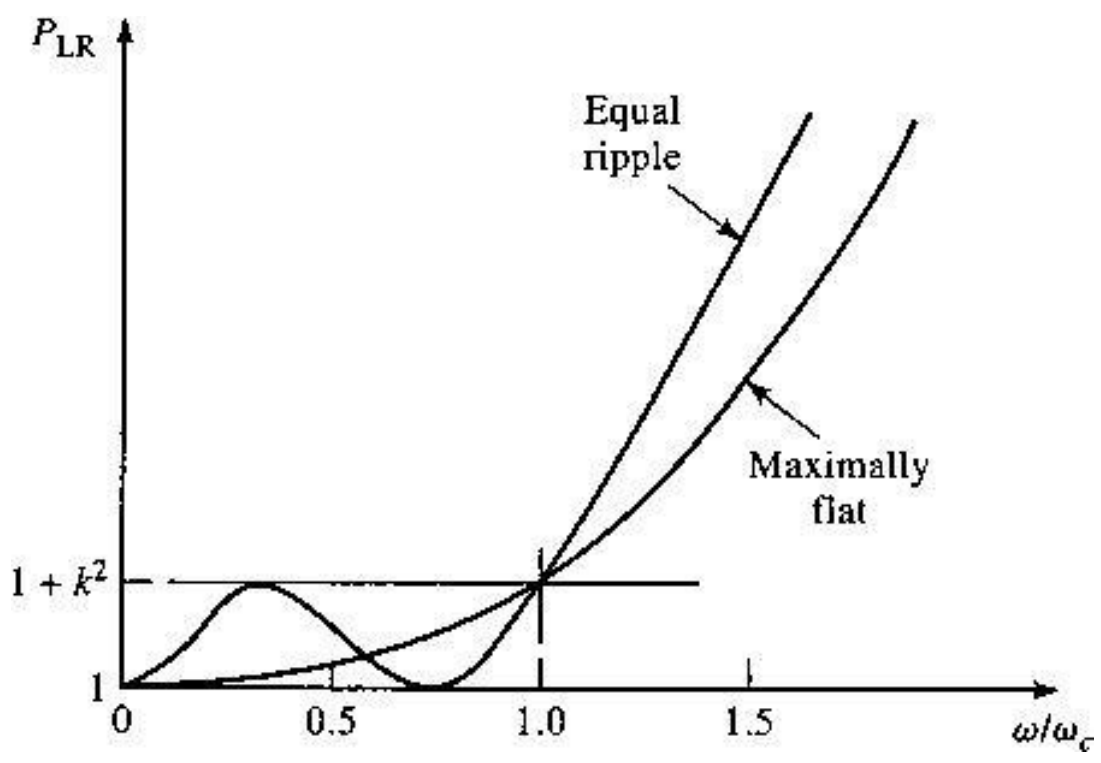

Fig.4. Equal-ripple versus maximally-flat response

The power loss ratio $\mathrm{P}_{\mathrm{LR}}$ is defined to be the ratio of the power available from source feeding the input port of the filter and power delivered to the load connected to the output port of the filter. The insertion loss (IL) of the filter (in $\mathrm{dB}$ ) is defined by the following equation.

$$
I L(d B)=10 \log P_{L R}
$$

The operating angular frequency $\omega$ in radians/second is given by

$$
\omega=2 \pi f
$$

where $\mathrm{f}$ is the operating frequency in Hz. Similarly, the cutoff angular frequency of the filter, $\omega_{\mathrm{c}}$, is given by the following equation.

$$
\omega_{c}=2 \pi f_{c}
$$

where $f_{c}$ is the cutoff frequency of the filter.

The cutoff frequency is defined as the value of operating frequency $f$ at which power loss ratio (in other words, insertion loss) goes above a predefined value. A popular choice for the design parameter k in Fig. 4 is $\mathrm{k}=1$. This yields $\mathrm{P}_{\mathrm{LR}}=2$ and $\mathrm{IL}=3 \mathrm{~dB}$ approximately. In case of this choice, the cutoff frequency is known as 3$\mathrm{dB}$ cutoff frequency.

The mathematical equation describing the dependence of insertion loss of a maximally-flat LPF on frequency is given below.

$$
I L=10 \log \left[1+\left(\frac{\omega}{\omega_{c}}\right)^{2 N}\right]
$$


In (37), IL is the insertion loss in $\mathrm{dB}$ at an angular stopband frequency $\omega, \omega_{\mathrm{c}}$ is the angular cutoff frequency, and $\mathrm{N}$ is the order of the filter.

The mathematical equation describing the dependence of insertion loss of an equal-ripple LPF on frequency is given below.

$$
I L=10 \log \left[1+T_{N} 2\left(\frac{\omega}{\omega_{c}}\right)\right]
$$

In (38), IL is the insertion loss in $\mathrm{dB}$ at an angular stopband frequency $\omega, \omega_{\mathrm{c}}$ is the angular cutoff frequency, and $\mathrm{N}$ is the order of the filter. $\mathrm{T}_{\mathrm{N}}(\mathrm{x})$ represents a Chebyshev polynomial of order $\mathrm{N}$ and argument $\mathrm{x}$.

Two prototype 'ladder' networks, shown in Fig. 5, are normally employed to design a LPF with lumped components (inductors and capacitors).

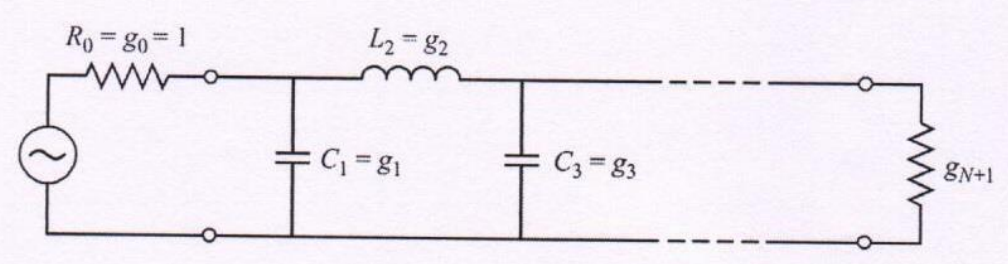

(a)

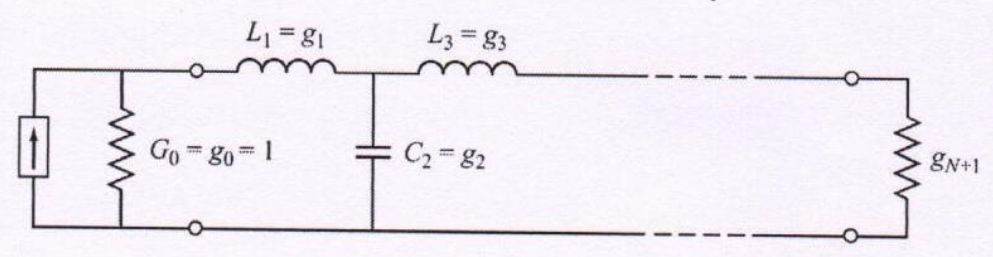

(b)

Fig.5. LPF prototypes (a) prototype beginning with a shunt element (b) prototype beginning with a series element

One can use either a prototype beginning with a parallel capacitor or a prototype beginning with a series inductor. In both cases, we first design the filter assuming that the source resistance $\mathrm{R}_{0}$ is unity and the angular cutoff frequency $\omega_{c}$ is unity. First, the order of the filter (indicating the total number of components) is determined using either (37) or (38), or using the graphs shown in Fig. 6 (for maximally-flat design) and Fig.7 (for equal-ripple design). The value of filter order can be directly read from the graph if the minimum required value of insertion loss at a specified stopband frequency is given. Once the order is determined, we use either Table 1 or Table 2 to read the component values ( $g$ values) for the basic prototype $\left(\omega_{c}=1, g_{0}=1\right)$. Then the design is impedance-scaled by using the following equations [7].

$$
\begin{aligned}
& L_{k}(\text { new })=R_{0} L_{k}(\text { old }) \\
& C_{k}(\text { new })=C_{k}(\text { old }) / R_{0}
\end{aligned}
$$

where $\mathrm{L}_{\mathrm{k}}$ (new) and $\mathrm{C}_{\mathrm{k}}$ (new) are the component values obtained after impedance-scaling, and $\mathrm{L}_{\mathrm{k}}(\mathrm{old})$ and $\mathrm{C}_{\mathrm{k}}(\mathrm{old})$ are the component values read from Table 1 or Table $2 . R_{0}$ is the actual value of the source impedance. 
Finally, the design is frequency-scaled to the actual $\omega_{\mathrm{c}}$ value by using the following equations.

$$
\begin{aligned}
& L_{k^{\prime}}=L_{k} /\left(2 \pi f_{c}\right) \\
& C_{k^{\prime}}=C_{k} /\left(2 \pi f_{c}\right)
\end{aligned}
$$

where $\mathrm{L}_{\mathrm{k}}$ and $\mathrm{C}_{\mathrm{k}}$ are the element values obtained after impedance-scaling, and $\mathrm{L}_{\mathrm{k}}$, and $\mathrm{C}_{\mathrm{k}}$, are the new element values obtained after frequency-scaling the design.

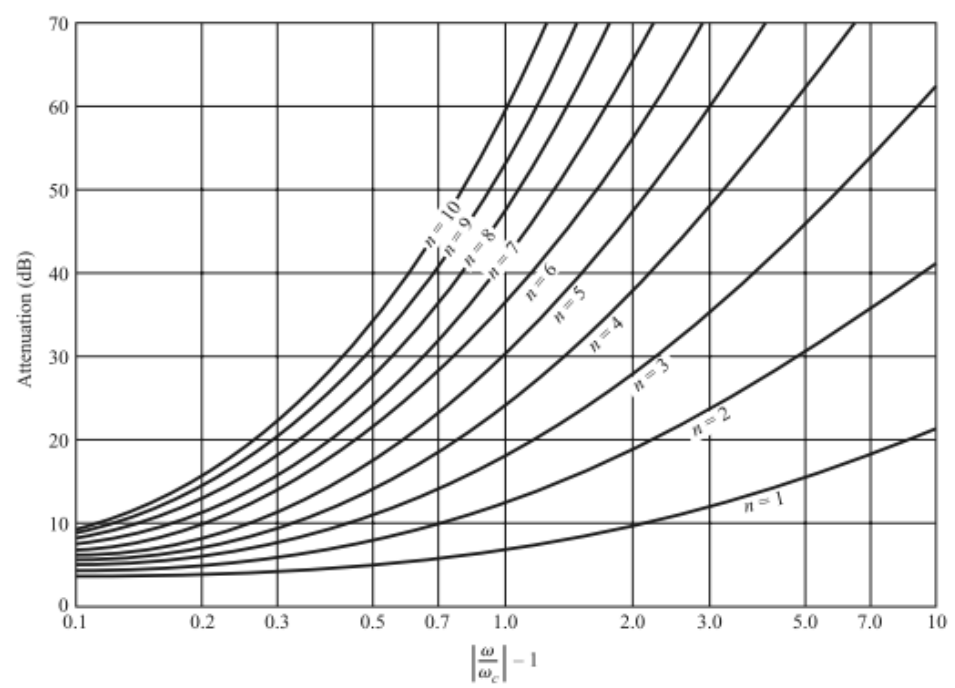

Fig.6. Graph used for determining the order of the maximally-flat LPF prototype

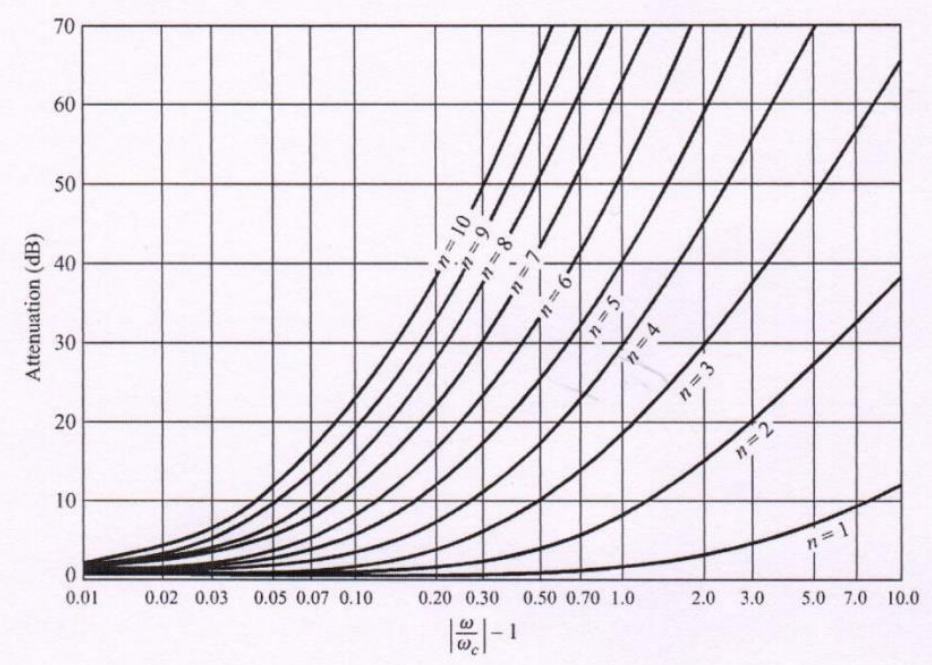

Fig.7. Graph used for determining the order of the equal-ripple LPF prototype (assuming $0.5 \mathrm{~dB}$ ripple) 
Table 1. Element values $\mathrm{g}_{\mathrm{k}}$ for a maximally-flat LPF prototype for $\mathrm{N}=1$ to $10\left(\mathrm{~g}_{0}=1, \omega_{\mathrm{c}}=1\right)$

\begin{tabular}{|c|c|c|c|c|c|c|c|c|c|c|c|}
\hline $\mathrm{N}$ & $\mathrm{k}=1$ & $\mathrm{k}=2$ & $\mathrm{k}=3$ & $\mathrm{k}=4$ & $\mathrm{k}=5$ & $\mathrm{k}=6$ & $\mathrm{k}=7$ & $\mathrm{k}=8$ & $\mathrm{k}=9$ & $\mathrm{k}=10$ & $\mathrm{k}=11$ \\
\hline 1 & 2.0000 & 1.0000 & & & & & & & & & \\
\hline 2 & 1.4142 & 1.4142 & 1,0000 & & & & & & & & \\
\hline 3 & 1.0000 & 2.0000 & 1.0000 & 1.0000 & & & & & & & \\
\hline 4 & 0.7654 & 1.8478 & 1.8478 & 0.7654 & 1.0000 & & & & & & \\
\hline 5 & 0.6180 & 1.6180 & 2.0000 & 1.6180 & 0.6180 & 1.0000 & & & & & \\
\hline 6 & 0.5176 & 1.4142 & 1.9318 & 1.9318 & 1.4142 & 0.5176 & 1.0000 & & & & \\
\hline 7 & 0.4450 & 1.2470 & 1.8019 & 2.0000 & 1.8019 & 1.2470 & 0.4450 & 1.0000 & & & \\
\hline 8 & 0.3902 & 1.1111 & 1.6629 & 1.9615 & 1.9615 & 1.6629 & 1.1111 & 0.3902 & 1.0000 & & \\
\hline 9 & 0.3473 & 1.0000 & 1.5321 & 1.8794 & 2.0000 & 1.8794 & 1.5321 & 1.0000 & 0.3473 & 1.0000 & \\
\hline 10 & 0.3129 & 0.9080 & 1.4142 & 1.7820 & 1.9754 & 1.9754 & 1.7820 & 1.4142 & 0.9080 & 0.3129 & 1.0000 \\
\hline
\end{tabular}

Table 2. Element values $\mathrm{g}_{\mathrm{k}}$ for an equal-ripple LPF prototype for $\mathrm{N}=1$ to $10\left(\mathrm{~g}_{0}=1, \omega_{\mathrm{c}}=1,0.5 \mathrm{~dB}\right.$ ripple)

\begin{tabular}{|c|c|c|c|c|c|c|c|c|c|c|c|}
\hline $\mathrm{N}$ & $\mathrm{k}=1$ & $\mathrm{k}=2$ & $\mathrm{k}=3$ & $\mathrm{k}=4$ & $\mathrm{k}=5$ & $\mathrm{k}=6$ & $\mathrm{k}=7$ & $\mathrm{k}=8$ & $\mathrm{k}=9$ & $\mathrm{k}=10$ & $\mathrm{k}=11$ \\
\hline 1 & 0.6986 & 1.0000 & & & & & & & & & \\
\hline 2 & 1.4029 & 0.7071 & 1.9841 & & & & & & & & \\
\hline 3 & 1.5963 & 1.0967 & 1.5963 & 1.0000 & & & & & & & \\
\hline 4 & 1.6703 & 1.1926 & 2.3661 & 0.8419 & 1.9841 & & & & & & \\
\hline 5 & 1.7058 & 1.2296 & 2.5408 & 1.2296 & 1.7058 & 1.0000 & & & & & \\
\hline 6 & 1.7254 & 1.2479 & 2.6064 & 1.3137 & 2.4758 & 0.8696 & 1.9841 & & & & \\
\hline 7 & 1.7372 & 1.2583 & 2.6381 & 1.3444 & 2.6381 & 1.2583 & 1.7372 & 1.0000 & & & \\
\hline 8 & 1.7451 & 1.2647 & 2.6564 & 1.3590 & 2.6964 & 1.3389 & 2.5093 & 0.8796 & 1.9841 & & \\
\hline 9 & 1.7504 & 1.2690 & 2.6678 & 1.3673 & 2.7239 & 1.3673 & 2.6678 & 1.2690 & 1.7504 & 1.0000 & \\
\hline 10 & 1.7543 & 1.2721 & 2.6754 & 1.3725 & 2.7392 & 1.3806 & 2.7231 & 1.3485 & 2.5239 & 0.8842 & 1.9841 \\
\hline
\end{tabular}

\subsection{Highpass Filter (HPF) Design, Bandpass Filter (BPF) Design, and Bandstop Filter Design}

To accomplish highpass, bandpass, or bandstop filter designs, the use of suitable transformations (LPF to HPF, LPF to BPF, etc.) is made. Detailed equations and procedures for doing so are available in literature [2], [7], [10].

\section{RF/Microwave Filter Design using Microstrips}

The lumped-component method of designing electronic filters, discussed in section 3, starts failing in radio frequency (RF) and microwave frequency bands since the availability of lumped components (inductors, capacitors, and resistors) starts becoming a major issue. The use of 'distributed' components becomes an attractive option. Inductors and capacitors are realized using sections of transmission lines in novel ways [1][17]. Microstrip line fits well in this category, since it is an easy-to-use transmission line, which can lead to highly compact, cost-effective designs.

A brief overview of the three most commonly used microstrip filters considered in the present work is presented below, 


\subsection{Stepped-Impedance Resonator Lowpass Filter (SIR LPF)}

A simple method of realizing microstrip lowpass filters, shown in Fig. 8 for a seventh-order filter, is to use a cascaded chain of microstrips, with alternating sections of high and low characteristic impedance values. The high characteristic impedance value $\mathrm{Z}_{\mathrm{OH}}$ is typically chosen to be between $100 \Omega$ and $130 \Omega$. The low characteristic impedance value $\mathrm{Z}_{\mathrm{OL}}$ is typically chosen to be between $10 \Omega$ and $20 \Omega$. Such filters are known as stepped-impedance resonator (SIR) filters, or high-Z, low-Z filters.

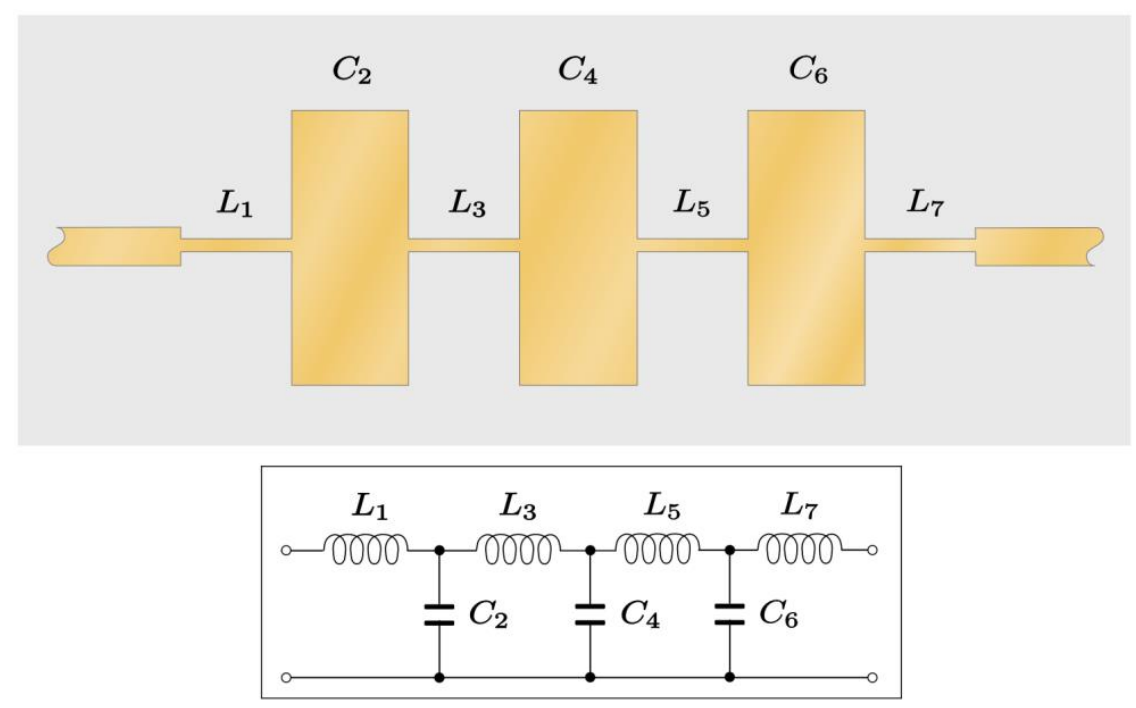

Fig.8. The schematic of a stepped-impedance resonator lowpass filter

As shown in Fig. 8, wider microstrips (having low characteristic impedance) represent parallel capacitors and narrower microstrips (having high characteristic impedance) represent series inductors. The design procedure consists essentially of three steps. The first step is to obtain, based on the given design specifications, a lumped-component design using the procedure described in section 3 . The second step is to convert the required $\mathrm{L}$ and $\mathrm{C}$ values into equivalent narrow and wide microstrips using the procedure given below.

Let $\mathrm{Z}_{\mathrm{OL}}$ be the characteristic impedance for low-impedance line and $\mathrm{Z}_{\mathrm{OH}}$ be the characteristic impedance for high-impedance line. Assuming that the substrate height and substrate dielectric constant are known, (1) - (9) can be used to calculate strip widths and $\beta$ values for each section of the filter. The electrical lengths of inductor sections can then be determined using

$$
\beta l=\frac{L R_{o}}{Z_{O H}}
$$

and the electrical lengths of capacitor sections can be determined using

$$
\beta l=\frac{C Z_{O L}}{R_{o}}
$$


where $\mathrm{L}$ and $\mathrm{C}$ are the normalized element values of the lowpass prototype filter. Once electrical lengths are known, physical lengths can easily be determined.

After the 'first-cut' design has been obtained using the procedure outlined above, the design can be further analyzed and optimized by writing a MATLAB program for calculating the individual ABCD parameters of each section, and then multiplying the individual ABCD-matrices to get the overall ABCD-matrix. Once the overall $\mathrm{ABCD}$-matrix is obtained, the insertion loss versus frequency response and return loss versus frequency response can be computed using equations presented in section 2.

Besides using MATLAB, one can also analyze/optimize the design using commercially available specialized simulation tools like ANSOFT, HFSS, IE3D, and ADS [18]-[20]. However, commercially available tools are quite expensive to purchase and maintain, which gives MATLAB-based tools a clear edge over them.

\subsection{Parallel-Coupled Bandpass Filter Design}

Fig. 9 shows the general structure of a fourth-order parallel-coupled microstrip bandpass filter. This type of design cascades several coupled-sections of microstrips to obtain a desired bandpass response. Each coupledsection is half-wave long. However, only quarter-wave portion of each section is coupled to the next section.

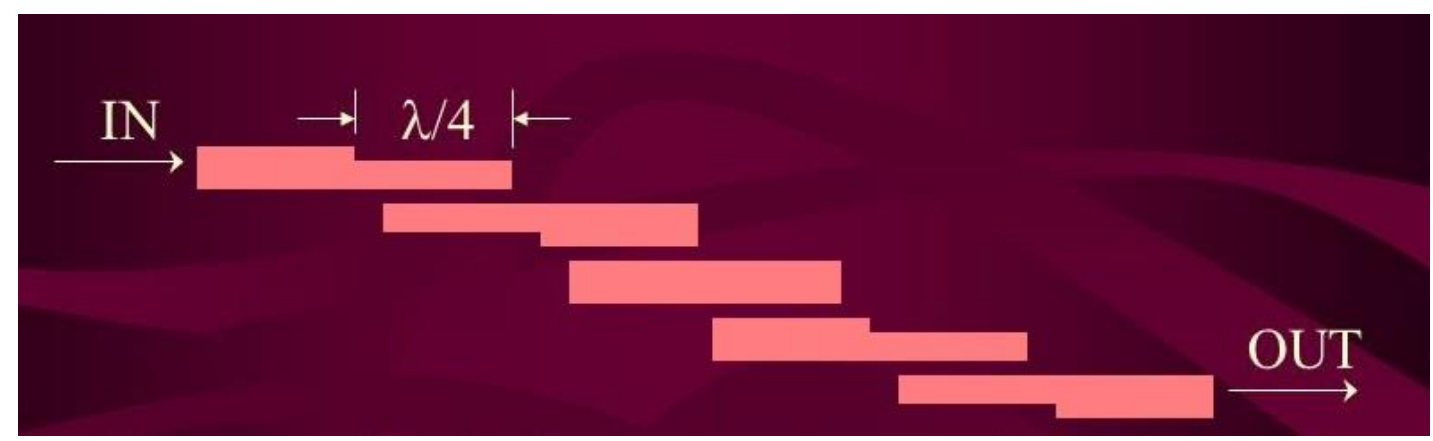

Fig.9. General structure of a parallel-coupled microstrip filter

Fig. 10 shows the schematic of a N-section parallel-coupled filter. An even-mode impedance Zoe and an odd-mode impedance Zoo, as shown in the figure characterize each section. The main advantage of such a filter is that it provides a wider bandwidth compared to end-coupled bandpass filter (discussed in sub-section 4.3.

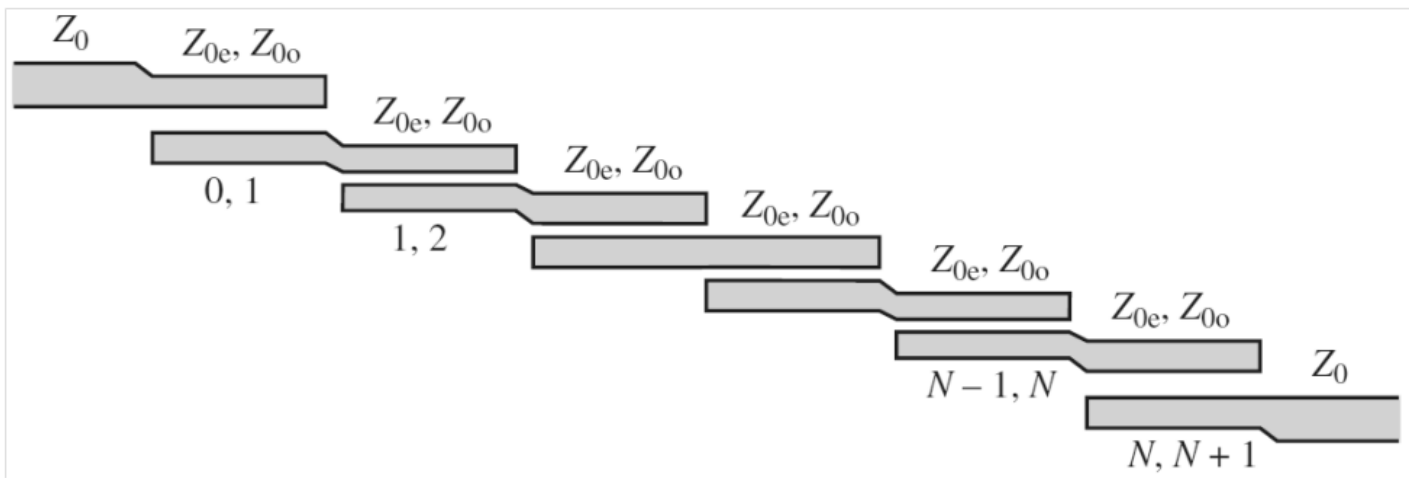

Fig.10. The schematic of an N-section parallel-coupled microstrip filter 
The first step in the design of such filters is to design the corresponding LPF prototype, assuming that the filter order and the nature of response (maximally-flat or equal-ripple) is given. The second step is to use the following equations for calculating admittance inverter values, $\mathrm{J}_{\mathrm{n}}$, for all sections [7], [10].

$$
\begin{aligned}
& \frac{J_{01}}{Y_{o}}=\sqrt{\frac{\pi F B W}{2 g_{0} g_{1}}} \\
& \frac{J j_{j+1}}{Y_{o}}=\frac{\pi F B W}{2} \frac{1}{\sqrt{g_{j} g_{j+1}}} \\
& \frac{J_{n, n+1}}{Y_{o}}=\sqrt{\frac{\pi F B W}{2 g_{n} g_{n+1}}}
\end{aligned}
$$

where FBW is the fractional bandwidth of the filter which is given by

$$
F B W=\frac{\omega_{2}-\omega_{1}}{\omega_{o}}
$$

In (48), $\omega_{1}$ and $\omega_{2}$ are the passband-edge angular frequencies and $\omega_{0}$ is the center angular frequency. $Y_{0}$ is the characteristic admittance, which is given by

$$
Y_{o}=\frac{1}{Z_{o}}
$$

The third step is to calculate even-mode and odd-mode line impedances for each section by using the following equations.

$$
\begin{aligned}
& Z_{O e}=Z_{o}\left[1+J Z_{o}+\left(J Z_{o}\right)^{2}\right] \\
& Z_{O o}=Z_{o}\left[1-J Z_{o}+\left(J Z_{o}\right)^{2}\right]
\end{aligned}
$$

The final step is to compute the line widths and line spacing using the following equations.

$$
\begin{aligned}
& \frac{s}{h}=\frac{2}{\pi} \cosh ^{-1}\left[\frac{\cosh \left(\left(\frac{\pi}{2}\right)\left(\frac{W}{h}\right)_{s e}\right)+\cosh \left(\left(\frac{\pi}{2}\right)\left(\frac{W}{h}\right)_{s o}\right)-2}{\cosh \left(\left(\frac{\pi}{2}\right)\left(\frac{W}{h}\right)_{s o}\right)-\cosh \left(\left(\frac{\pi}{2}\right)\left(\frac{W}{h}\right)_{s e}\right)}\right] \\
& \frac{w}{h}=\frac{1}{\pi}\left[\cosh ^{-1}\left(\frac{1}{2}\left[\left(\cosh \left(\frac{\pi s}{2 h}\right)-1\right)+\left(\cosh \left(\frac{\pi s}{2 h}\right)+1\right) \cosh \left(\left(\frac{\pi}{2}\right)\left(\frac{W}{h}\right)_{s e}\right)\right]\right)-\left(\frac{\pi}{2}\right)\left(\frac{s}{h}\right)\right]
\end{aligned}
$$


After the 'first-cut' design has been obtained using the procedure outlined above, the design can be further analyzed and optimized by writing a MATLAB program for calculating the individual ABCD parameters of each coupled section, and then multiplying the individual ABCD-matrices to get the overall ABCD-matrix. Once the overall ABCD-matrix is obtained, the insertion loss versus frequency response and return loss versus frequency response of the designed filter can be computed using equations presented in section 2 .

Besides using MATLAB, one can also analyze/optimize the design using commercially available specialized simulation tools like ANSOFT, HFSS, IE3D, and ADS [18]-[20]. However, commercially available tools are quite expensive to purchase and maintain, which gives MATLAB-based tools a clear edge over them.

\subsection{End-Coupled Bandpass Filter Design}

The end-coupled bandpass microstrip filter consists of a series of microstrip lines of equal widths, with air gap between them, as shown in Fig. 11. The air gaps can be modeled using a series capacitor [7], [10]. The filter can then be modeled as a series of capacitive coupled half-wave resonators. The electrical lengths of the half-wavelength resonators attributed to the J-inverters are determined by

$$
\theta_{i}=\pi-\frac{1}{2}\left[\tan ^{-1}\left(2 Z_{o} B_{i}\right)+\tan ^{-1}\left(2 Z_{o} B_{i+1}\right)\right]
$$

where B is the capacitive susceptance given by

$$
B_{i}=\frac{J_{i}}{1-\left(Z_{o} J_{i}\right)^{2}}
$$

Once the electrical lengths are obtained, the closed-form mathematical expressions available in [7] can be used to calculate the coupling gap and physical lengths of the microstrip sections.

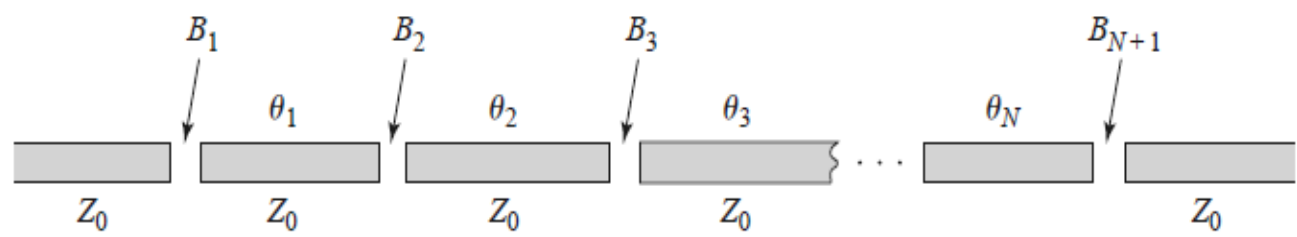

Fig.11. An N-section end-coupled microstrip filter

After the 'first-cut' design has been obtained using the procedure outlined above, the design can be further analyzed and optimized by writing a MATLAB program for calculating the individual ABCD parameters of each section (including gaps), and then multiplying the individual ABCD-matrices to get the overall ABCDmatrix. Once the overall ABCD-matrix is obtained, the insertion loss versus frequency response and return loss versus frequency response of the designed filter can be computed using equations presented in section 2 .

Besides using MATLAB, one can also analyze/optimize the design using commercially available specialized simulation tools like ANSOFT, HFSS, IE3D, and ADS [18]-[20]. However, commercially available tools are quite expensive to purchase and maintain, which gives MATLAB-based tools a clear edge over them. 


\section{Description of the MATLAB Tool}

Based on design equations and procedures presented in sections 2, 3, and 4, a MATLAB tool was developed to design and analyze three types of microstrip filters, namely, stepped-impedance resonator filter, parallelcoupled filter, and end-coupled filter. The MATLAB code for this tool is available upon request.

The tool has been designed to have an easy-to-use user interface (UI). The first interface is shown in Fig. 12. This interface allows the user to choose the type of filer to be designed. By clicking on the filter type, an appropriate new window pops up as shown in Figs. 13-15.

Select a Filter to continue

Low Pass Filter

End Coupled BandPass Filter

Parallel Coupled BandPass Filter

Exit

Fig.12. First interface of the MATLAB design tool

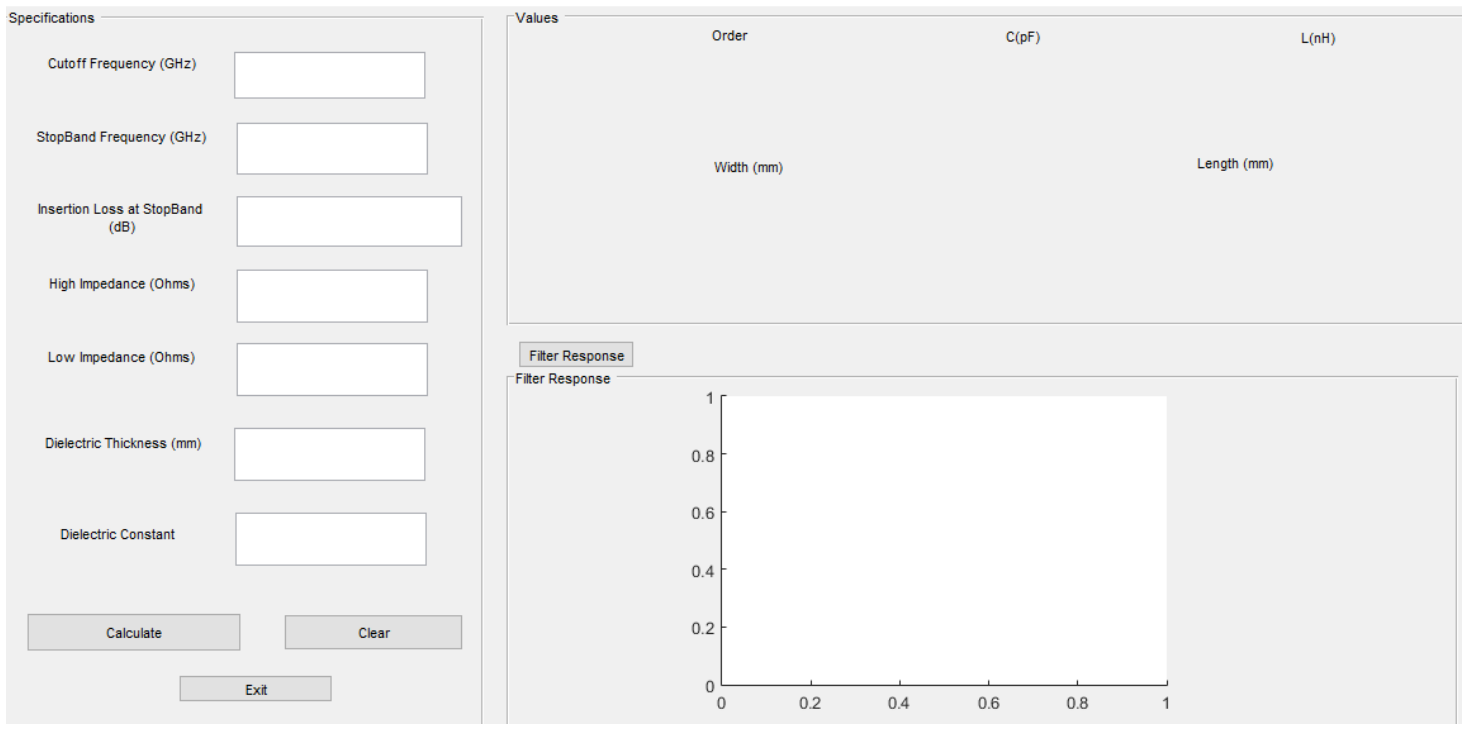

Fig.13. Stepped-impedance resonator lowpass filter interface of the MATLAB design tool 


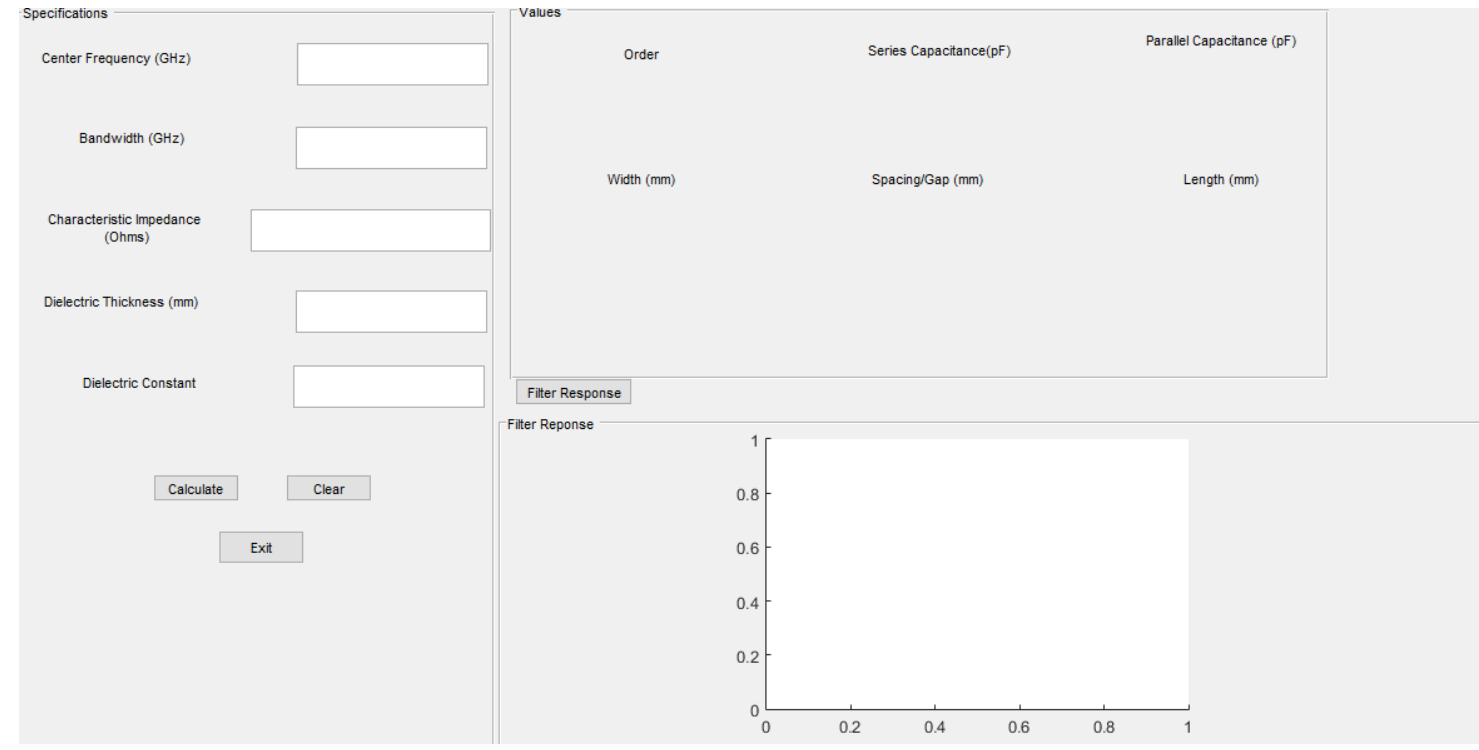

Fig.14. End-coupled bandpass filter interface of the MATLAB design tool

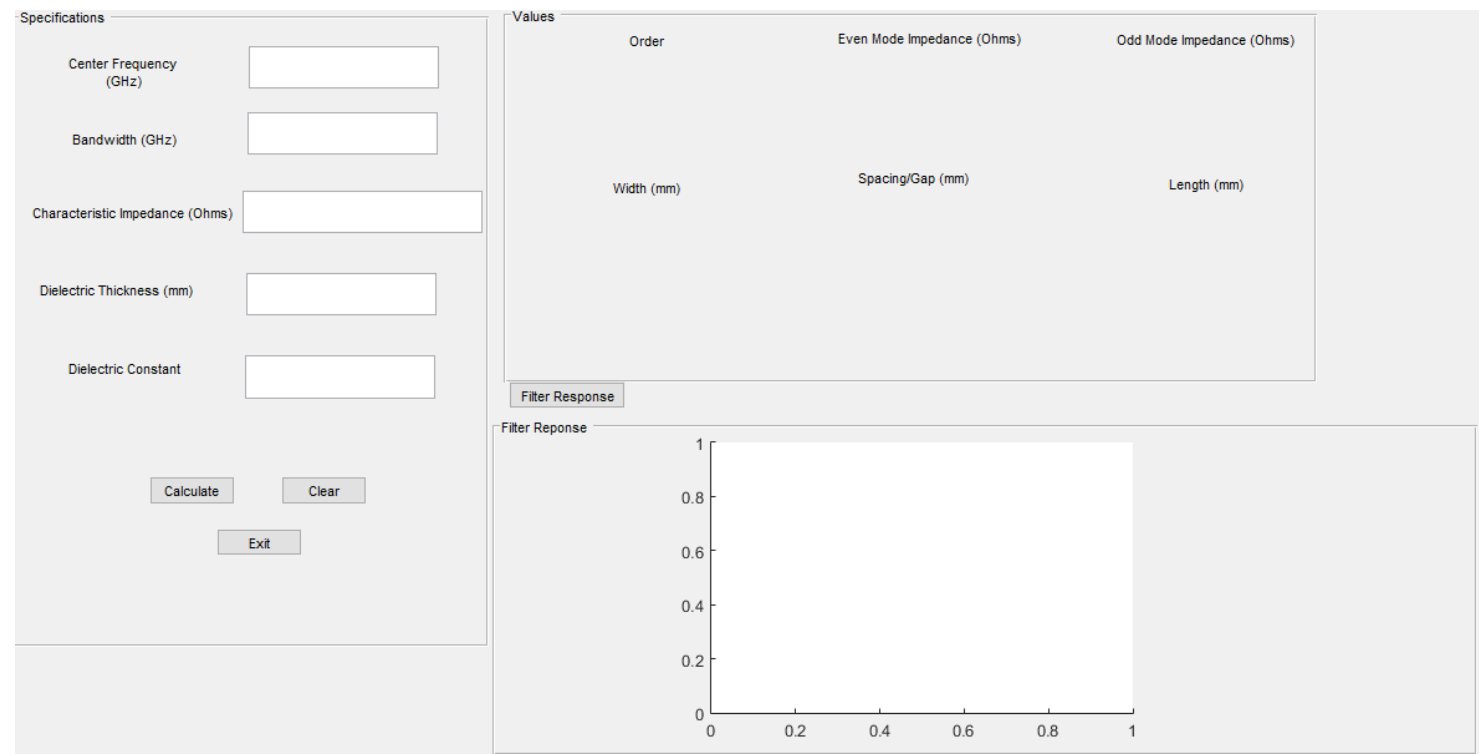

Fig.15. Parallel-coupled bandpass filter interface of the MATLAB design tool

For the stepped-impedance resonator lowpass filter, the specifications that need to be provided by the user are cutoff frequency, minimum insertion loss at a given stopband frequency, characteristic impedance of narrow sections, characteristic impedance of wide sections, substrate thickness, and the value of the dielectric constant of the substrate. Then the 'Calculate' button is clicked. The tool then shows the filter order required, capacitance values, inductance values, microstrip widths, microstrip lengths, and the predicted response of the filter. 'Clear' button resets the input values. 'Exit' button closes the window. 
For the end-coupled resonator bandpass filter, the specifications that need to be provided by the user are center frequency, characteristic impedance of the microstrip, substrate thickness, and the value of the dielectric constant of the substrate. Then the 'Calculate' button is clicked. The tool then shows the filter order value, microstrip widths, microstrip lengths, spacing value, and the predicted response of the filter. 'Clear' button resets the input values. 'Exit' button closes the window.

For the parallel-coupled bandpass filter, the specifications that need to be provided by the user are center frequency, characteristic impedance of the microstrip, substrate thickness, and the value of the dielectric constant of the substrate. Then the 'Calculate' button is clicked. The tool then shows the filter order value, microstrip widths, microstrip lengths, spacing values, and the predicted response of the filter. 'Clear' button resets the input values. 'Exit' button closes the window.

\section{Results}

The accuracy of the tool was verified through using it for a large variety of design examples. A detailed comparison was made against data obtained using Ansoft simulator and the agreement was, in general, found to be quite good. Two sample design examples are briefly discussed below.

\subsection{Design Example 1: A Stepped-Impedance Resonator Lowpass Filter}

A stepped-impedance resonator lowpass filter was designed with the following specifications:

1. 3-dB cutoff frequency

2. Insertion loss at $4 \mathrm{GHz}$

3. High impedance value

4. Low impedance value

5. Dielectric thickness

6. Dielectric constant

7. Input/output impedance
$2.4 \mathrm{GHz}$

$20 \mathrm{~dB}$ minimum

$120 \Omega$

$20 \Omega$

$1.58 \mathrm{~mm}$

4.4 (FR4 substrate)

The results obtained using our MATLAB tool are shown in Fig. 16.

The response shows an insertion loss value of approximately $4.06 \mathrm{~dB}$ at the 3 - $\mathrm{dB}$ cutoff frequency. When compared with data obtained through implementing the same design in Ansoft, the result was seen to be approximately $4.25 \mathrm{~dB}$. The response shows an insertion loss value of approximately $22.77 \mathrm{~dB}$ at $4 \mathrm{GHz}$ frequency, whereas the insertion loss value given by Ansoft tool was approximately $23.16 \mathrm{~dB}$. The rest of the S21 curve obtained using MATLAB tool matched well with that obtained using Ansoft tool.

\subsection{Design Example 2: An End-Coupled Bandpass Filter}

An end-coupled bandpass filter was designed with the following specifications:

1. Center frequency

2. Bandwidth

3. Characteristic impedance

4. Dielectric constant

5. Dielectric thickness
$6 \mathrm{GHz}$

$0.25 \mathrm{GHz}$

$50 \Omega$

9.8 (Alumina)

$1.58 \mathrm{~mm}$ 

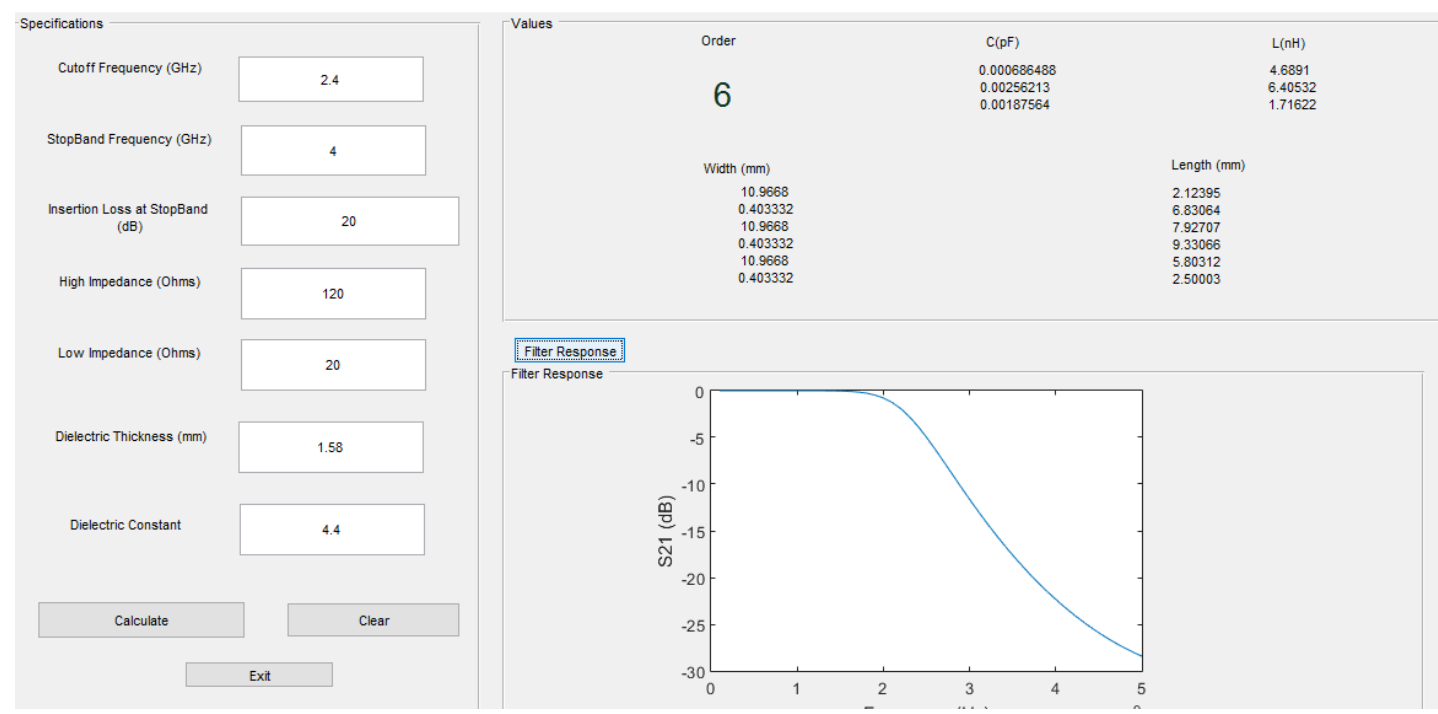

Fig.16. Design example 1 results obtained using the MATLAB design tool

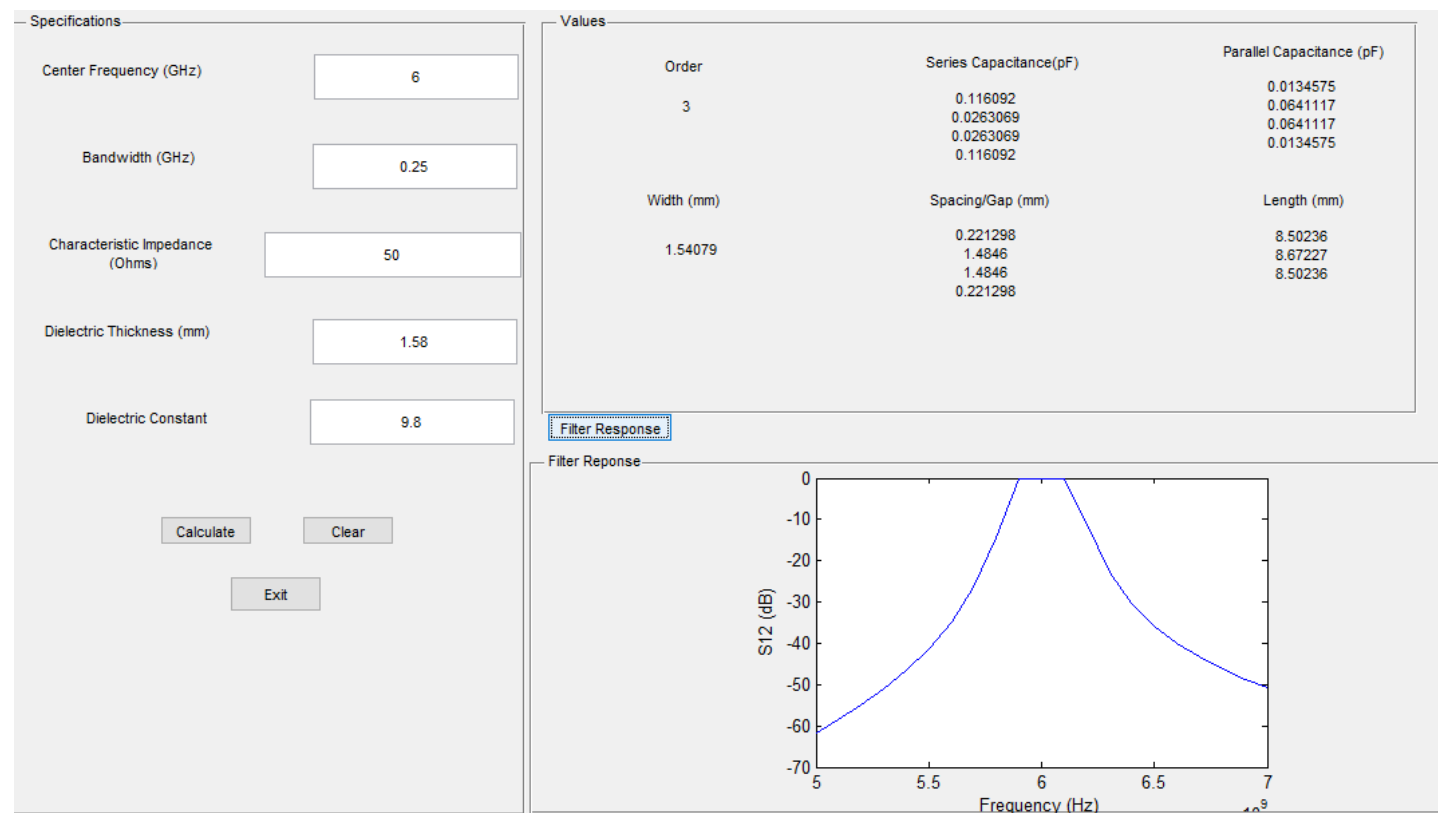

Fig.17. Design example 2 results obtained using the MATLAB design tool

The results obtained using our MATLAB tool are shown in Fig. 17.The agreement with Ansoft results was again found to be quite good. For instance, the passband insertion loss was approximately equal to $1.33 \mathrm{~dB}$ at 6 $\mathrm{GHz}$ when using the MATLAB tool, and was approximately equal to $1.98 \mathrm{~dB}$ when using the ANSOFT tool. The insertion loss at $5.75 \mathrm{GHz}$ was approximately equal to $19.5 \mathrm{~dB}$ while using the MATLAB tool, and was approximately equal to $18.75 \mathrm{~dB}$ while using the ANSOFT tool. 


\section{Conclusions}

A new MATLAB based user-friendly interactive design tool for microstrip filters is reported. The accuracy of the results produced by the tool has been found to be comparable to that obtained using expensive commercially- available tools of this type. Only the stepped-impedance resonator lowpass filer, the endcoupled bandpass filter, and the parallel-coupled bandpass filter have been considered in the present version of the tool. The approach presented, however, is quite generic and is extendable to other types of microstrip filters too.

\section{References}

[1] Zhou C, Guo P, Wu W. Compact UWB BPF with a tunable notched band based on triple-mode HMSIW resonator. Int. J. Wireless Microwave Technologies 2016; 5:901-7.

[2] Ahmad BH, Mazlan MH, Husain MN, Zakaria Z, Shairi NA. Microstrip filter design techniques: an overview. ARPN J. Engg. Appl. Sc. 2015; 2:1-9.

[3] Kushwah V, Bhadauria S, Tomar G. Design of microstrip low pass filter with defected ground structure. IEEE Int. Conf. Computational Intelligence and Communication Networks 2015.

[4] Tomar L, Gupta S, Tomar R, Bhartia P. MATLAB-based computer-aided-design algorithm for designing stepped-impedance-resonator low-pass filters in microstrip technology. Int. J. Wireless Microwave Technologies 2015; 1:1-20.

[5] Tomar L, Gupta S, Tomar R, Bhartia P. Design and analysis of low pass microstrip filters using MATLAB. IEEE $16^{\text {th }}$ Int. Symp. Quality Electronic Design 2015; 263-66.

[6] Singh P, Tomar R. The use of defected ground structures in designing microstrip filters with enhanced performance characteristics. Procedia Technology (Elsevier) 2014;17: 58-64.

[7] Pozar D. Microwave Engineering. $4^{\text {th }}$ ed. New York: Wiley; 2012.

[8] Das S, Chowdhury S. Design, simulation and fabrication of stepped impedance microstripline low pass filter for S-band applications using IE3D and MATLAB. Int. J. Electronics Commun. Engg. 2012; 1:98100.

[9] Guo L, Yu Z, Zhang Y. A dual-band band-pass filter using stepped impedance resonator. Microwave and Optical Technology Letters. 2011; 1:123-25.

[10] Hong J, Lancaster MJ. Microstrip filters for RF/microwave applications. $2^{\text {nd }}$ ed. New York: Wiley; 2011.

[11] Kaveri S. Design of tunable edge-coupled microstrip bandpass filters. MS Thesis, Utah State Univ. 2008.

[12] Mondal P, Mandal MK. Design of dual-band bandpass filters using stub-loaded open-loop resonators. IEEE Trans. Mic. Th. Tech. 2008; 1:150-55.

[13] Kuo J, Shih, E. Microstrip stepped impedance resonator bandpass filter with an extended optimal rejection bandwidth. IEEE Trans. Mic. Th. Tech. 2003; 5:1554-59.

[14] Fordham O, Ming-Ju T, Alexopoulos NG. Electromagnetic synthesis of overlap-gap-coupled microstrip filters. IEEE Int. Mic. Symp. 1995.

[15] Hill A, Burke J, Kottapalli K. Three-dimensional electromagnetic analysis of shielded microstrip circuits. Int. J. Microwave Millimeter Wave Computer Aided Engg. 1992; 4:286-96.

[16] Shibata T, Hayashi T, Kimura T. Analysis of microstrip circuits using three-dimensional full-wave electromagnetic field analysis in time domain. IEEE Trans. Mic. Th. Tech. 1988; 6:1064-70.

[17] Levy R, Cohn S. A history of microwave filter research, design, and development . IEEE Trans. Mic. Th. Tech. 1984; 9:1055-67.

[18] http://www.ansys.com/Products/Electronics/ANSYS-HFSS

[19] http://www.rfglobalnet.com/doc/zeland-ie3d-version-1212-features-rfid-antenn-0001

[20] http://www.keysight.com/en/pc-1297113/advanced-design-system-ads?cc=IN\&lc=eng 


\section{Authors' Profiles}

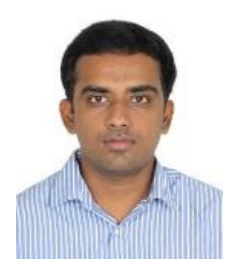

Shreyas Rao (born June 7, 1996) is a fourth year undergraduate student in the School of Electronics Engineering at VIT University, Chennai, India. He is passionate about designing RF circuits.

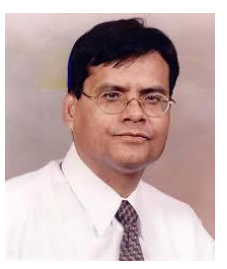

Raghuvir Tomar (born January 19, 1955) is a Professor of Electronics and Communication Engineering at The LNM Institute of Information Technology, Jaipur, India. He has approximately two decades of Industry experience in Canada and United States of America. He has co-authored more than sixty research papers and technical reports, and has coauthored a book on printed and microstrip antennas. His passion is to analyse and design $\mathrm{RF} /$ microwave systems and components.

How to cite this paper: Shreyas Rao, Raghuvir Tomar," A New MATLAB based Microstrip Filter Design Tool", International Journal of Wireless and Microwave Technologies(IJWMT), Vol.7, No.5, pp. 49-70, 2017.DOI: 10.5815/ijwmt.2017.05.06 